\title{
BMJ Open Value-based care pathway for inflammatory bowel disease: a protocol for the multicentre longitudinal non- randomised parallel cluster IBD Value study with baseline period
}

\author{
Reinier Cornelis Anthonius van Linschoten (D) , ${ }^{1,2}$ Nikki van Leeuwen, ${ }^{3}$ \\ Daan Nieboer, ${ }^{3}$ Erwin Birnie, ${ }^{4,5}$ Menne Scherpenzeel, ${ }^{6}$ Karen Evelyne Verweij, ${ }^{7}$ \\ Vincent de Jonge, ${ }^{8}$ Jan Antonius Hazelzet, ${ }^{3}$ C. Janneke van der Woude, ${ }^{2}$ \\ Rachel Louise West, ${ }^{1}$ Desirée van Noord, ${ }^{1}$ On behalf of the Southwest Netherlands \\ IBD Study Group
}

To cite: van Linschoten RCA, van Leeuwen N, Nieboer D, et al. Value-based care pathway for inflammatory bowel disease: a protocol for the multicentre longitudinal non-randomised parallel cluster IBD Value study with baseline period. BMJ Open 2022:12:e50539. doi:10.1136/ bmjopen-2021-050539

- Prepublication history and additional supplemental material for this paper are available online. To view these files, please visit the journal online (http://dx.doi.org/10.1136/ bmjopen-2021-050539).

RLW and DvN are joint senior authors.

Received 23 February 2021 Accepted 10 December 2021

\section{Check for updates}

(c) Author(s) (or their employer(s)) 2022. Re-use permitted under CC BY-NC. No commercial re-use. See rights and permissions. Published by BMJ.

For numbered affiliations see end of article.

\section{Correspondence to}

Reinier Cornelis Anthonius van Linschoten;

r.linschoten@franciscus.nl

\section{ABSTRACT}

Introduction Biologics are effective for the treatment of inflammatory bowel disease (IBD). However, unwarranted variation in processes and outcomes has been reported in the treatment of IBD. A care pathway for the treatment of IBD has the potential to reduce practice variation and improve outcomes. This study aims to compare the effect of a uniform care pathway for the treatment of patients with IBD with biologics to the current situation.

Methods and analysis IBD Value is a longitudinal multicentre non-randomised parallel cluster trial with a baseline period. The study takes place in eight centres in the Netherlands. The baseline period will run for 12 months, after which the care pathway will be implemented in 6 of the 8 participating hospitals during the implementation phase of 3 months. Hereafter, the effect of the care pathway will be assessed for 12 months. Total study period is 27 months. The primary outcome is the effect of the care pathway on disease control (IBD-Control questionnaire). Secondary outcomes are the effect of the care pathway on the other outcomes of the International Consortium of Health Outcomes Measurement IBD standard set, health-related generic quality of life, patient experiences and degree of variation; cost effectiveness of the care pathway; and the variation between hospitals in the aforementioned outcomes in the baseline period. Outcomes will be measured every 6 months. The study started on 1 December 2020 and a minimum of 200 patients will be included.

Ethics and dissemination The study was deemed not to be subject to Dutch law (WMO; Medical Research Involving Human Subjects Act) by the Medical Ethics Committee of the Erasmus MC, the Netherlands (registration number: MEC-2020-075) and a waiver was provided. Results will be disseminated through peer-reviewed journals and presented at (inter)national conferences.

Trial registration number NL8276.
Strength and limitation of the study

This study is, to the best of our knowledge, the first prospective multicentre study assessing the effect of a care pathway for the treatment of inflammatory bowel disease (IBD) on health outcomes.

- The use of a baseline period and control group allow for controlling for time trends when analysing the effect of the care pathway.

- The Dutch Crohn's and Colitis Patient Organisation was involved in the study design and will participate in the development of the care pathway.

- This is the first large multicentre study to implement the International Consortium of Health Outcomes Measurement standard set for IBD.

- As the study is a non-randomised trial, analyses will have to be adjusted for case mix to correct for possible confounding bias.

\section{INTRODUCTION}

Crohn's disease and ulcerative colitis, subtypes of inflammatory bowel disease (IBD), are chronic inflammatory diseases of the gastrointestinal tract. ${ }^{12}$ Signs and symptoms of IBD are abdominal pain, diarrhoea and rectal bleeding. IBD can also affect extraintestinal organs, such as the liver, skin, eyes and joints. ${ }^{3-5}$ Furthermore, IBD can have a major impact on quality of life because of fatigue and its psychological impact. ${ }^{6} 7$ To control these symptoms, patients are often dependent on medication and are sometimes hospitalised or need surgery when drugs fail. The high disease burden leads to reduced quality of life, high healthcare costs (between $€ 15000$ and $€ 30000$ per patient per year) and reduced work productivity. ${ }^{78}$ Biologics 
and new small molecules (e.g. tofacitinib) are proven efficacious treatments for IBD and have shown to induce and maintain remission, avert hospitalisation and surgery, and reduce productivity loss in randomised controlled trials. ${ }^{9-12}$

Considerable variation exists between healthcare providers in the treatment of IBD with biologics. ${ }^{13-18}$ Treatment variation consists, among other things, of differences in provided care and follow-up, such as type of medication prescribed, dosing frequency and interpretation of therapeutic drug monitoring. Treatment variation can lead to differences in outcomes, such as the proportion of patients in remission, side effects and treatment costs. ${ }^{19}$ While variation can be a natural consequence of differences between patient populations, a part of the variation in processes and outcomes was explained by experience and expertise of healthcare providers, with better process adherence and outcomes for dedicated IBD or academic physicians. ${ }^{17} 19$

Treatment variation might also lead to reduced effectiveness of biologics in daily practice. Observational population-based studies showed no association between the use of biologics and long-term disease progression, nor on hospitalisation or surgery, contradicting the findings of randomised controlled trials. ${ }^{20-22}$ Taking into account the differences in patient populations and study designs, these observational studies hypothesise that variation in treatment, mainly underuse and misuse of biologics, may partly explain the gap between the efficaciousness of biologics in randomised trials and their effectiveness in the real world. Reduction of this variation might thus be a potential avenue for improving outcomes of patients with IBD treated with a biologic.

Value-based healthcare (VBHC) is an approach that aims, among other things, at improving technical value (health outcomes achieved divided by resources spent) for the patient by tackling unwarranted variation and optimising the care delivery process. ${ }^{23}{ }^{24}$ Important parts of VBHC are systematically measuring both patientreported outcomes and the costs of achieving these outcomes. ${ }^{25}{ }^{26}$ These data can consequently be used to evaluate and adjust the care delivery process and improve (cost-)effectiveness of achieving optimal patient-centred outcomes.

Implementing a care pathway in clinical practice seems promising for improving value, which was illustrated by a retrospective pilot study that evaluated a care pathway for IBD in a VBHC programme. This care pathway showed a favourable effect on flares $(-26 \%)$ and costs $(-16 \%){ }^{27}$ Other studies supported the effect of a care pathway for IBD on costs and also showed an improvement of care processes. ${ }^{28}{ }^{29}$ In inguinal hernia repair, chronic heart failure and total hip replacement, the implementation of a care pathway, was also accompanied by reduced variation in processes and outcomes. ${ }^{30}$ Although these studies showed a promising effect on outcomes and processes, they suffered from low sample sizes, retrospective study designs and lacked patient-centred outcome measures.
With the prospective multicentre IBD Value study, we aim to assess the impact of a care pathway for the treatment of IBD with biologics and new small molecules on patientcentred outcomes.

\section{METHODS AND ANALYSIS}

The Standard Protocol Items: Recommendations for Interventional Trials guidelines were followed and the checklist is included with the protocol (online supplemental file 1). ${ }^{31}$ The most recent study protocol version 2.0.0 (July 2020) is presented in this manuscript. Changes to the protocol will be submitted to the Medical Ethics Committee Erasmus MC (Rotterdam, the Netherlands). Changes will also be noted in the trial register and communicated to local investigators. The start date of the study was 1 December 2020.

\section{Study aim}

The main objective of the study is to evaluate the added value of a uniform care pathway on the health outcomes of patients with IBD treated with a biologic or new small molecule in one of the participating hospitals. Secondary objectives are to:

- Assess the degree of regional variation in outcomes and costs of the treatment of IBD with biologics and new small molecules;

- Uncover areas of improvement in the care of patients with IBD

- Develop and implement a regional care pathway for the treatment of IBD with biologics and new small molecules based on scientific evidence, current guidelines and adapted to the local context;

- Evaluate the cost effectiveness of the care pathway.

- Evaluate the effect of the care pathway on variation in outcomes and costs.

\section{Study design}

This is a longitudinal multicentre non-randomised parallel cluster trial with a baseline period (figure 1). In the first 12 months of the study, before the introduction of the new care pathway, the current situation in IBD care for patients on biologics or new small molecules will be assessed in all participating hospitals to establish baseline measures. These data will primarily be used as comparison with the second study period after implementation of the care pathway. The data will also be used to

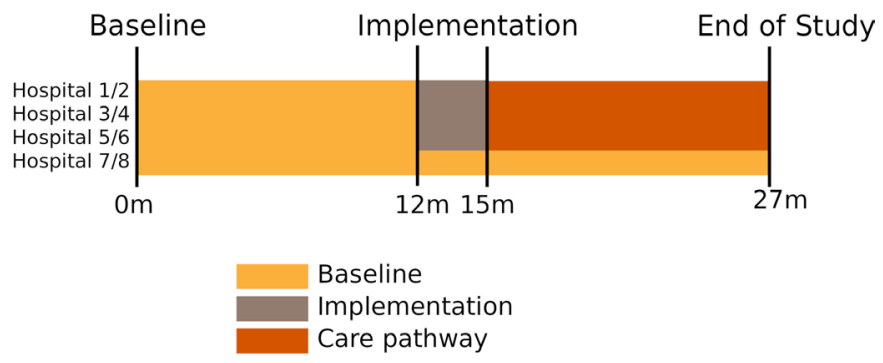

Figure 1 Study timeline. m, month. 
determine areas of improvement, as benchmarking, and aid the design of the care pathway. Subsequently, the care pathway will be implemented in six of the participating hospitals during a 3-month implementation period.

The participating hospitals are: Franciscus Gasthuis \& Vlietland, Rotterdam and Schiedam; Erasmus MC, Rotterdam; Albert Schweitzer Hospital, Dordrecht, Zwijndrecht and Sliedrecht; Maasstad Hospital, Rotterdam; Ikazia Hospital, Rotterdam; IJsselland Hospital, Capelle aan den IJssel; Reinier de Graaf Gasthuis, Delft; and Amphia Hospital, Breda. These are the hospitals that have collaborated in IBD BeterKeten in the southwest of the Netherlands since 2016 to improve quality of care of patients with IBD in the region. ${ }^{32}$ The care pathway will not be implemented in Reinier de Graaf Gasthuis and Amphia Hospital; these hospitals will participate as the control group. The content of the care pathway will only be revealed to and implemented in the six hospitals in the intervention group at the start of the implementation period. The development of the care pathway will be completed by the working group in the last period of the baseline measurement phase. After implementation, outcomes will be evaluated during the 12-month follow-up period in all participating hospitals.

\section{Population}

The study population comprises all patients with IBD being treated with a biologic or new small molecule in the eight participating hospitals. The care pathway also covers patients treated with new small molecules, as these belong to the same group as patients treated with a biologic: complex disease and a high cost of treatment. Approximately 3200 patients are treated with the aforementioned medication in these hospitals in total.

All participants will meet the following criteria:

- 18 years of age or older.

- Have given informed consent for data collection.

- Being treated for IBD in one of the participating hospitals.

- Have an IBD diagnosis of at least 3 months.

- Treated with one of the currently registered biologics or new small molecules for IBD treatment or new treatments registered during the study period, including infliximab, adalimumab, golimumab, vedolizumab, ustekinumab or tofacitinib.

A potential subject may be excluded from study participation if they have insufficient knowledge of the Dutch language to complete the questionnaires and/or have no access to the internet to complete the questionnaires.

\section{Intervention}

Design

The intervention is a uniform care pathway for the treatment of patients with IBD with a biologic or new small molecule. It contains uniform guidelines for prescribing, the work-up and switching of biologic therapy and new small molecules, and for the frequency and type of follow-up. As IBD is a heterogeneous disease, the care pathway will not be able to cover all possible treatment decisions but aims to guarantee the same level of care for patients with IBD in all participating hospitals, while taking into account patient preference and uncertainty in the evidence concerning IBD treatment.

To prevent contamination of the control period, the development of the care pathway will be finalised shortly before implementation. The care pathway will be developed by an IBD BeterKeten working group of gastroenterologists and IBD nurses with multidisciplinary input of a surgeon and a dietician. Moreover, the Dutch IBD patient federation (Crohn \& Colitis NL) will participate in the design of the care pathway. The care pathway will be based on national and international guidelines and will be designed according to the following steps. ${ }^{33-35}$

First, the main topics of what the care pathway should cover will be drafted by the project manager. These topics will then be discussed until consensus is reached by the working group. Hereafter, the project manager will draft care pathways for each topic (see the Content section) on the basis of (inter) national guidelines. These drafts will then be discussed in the working group until consensus is reached on exact content and timing of the care pathway. Literature searches will be performed to inform the working group in cases of uncertainty around best practices. When the evidence around treatment decisions is uncertain or scarce, this will be clearly reflected in the care pathway.

Outcomes from the baseline measurement collected during the first project phase will be used to adjust and improve the care pathway. These will be analysed according to their prespecified definitions (see the Outcome section) and stratified per institute to assess areas of improvement in IBD care. Results of these analyses and consequences for improvement will be discussed in a working group meeting and implemented in the care pathway. The final draft of the care pathway will be presented for approval of the IBD specialists of all participating intervention centres.

\section{Content}

The care pathway will address the following issues

1. Actions that do not depend on current treatment but apply to all patients: examples are periodical colorectal cancer and micronutrient screening.

2. Evaluation of a possible flare: when a patient presents with symptoms or when abnormal test results are found, differential diagnoses have to be excluded. Moreover, disease activity has to be measured using objective markers.

3. Therapy sequence in case of a flare: it will indicate advice on the next treatment step for a patient with a flare based on their disease and treatment history. This could be either treatment intensification or switching.

4. Frequency, type and timing of follow-up for the induction and remission phases of the different therapies: examples are the timing of outpatient clinic visits, laboratory assessments and additional examinations. 
The care pathway is a decision-making tool for care providers and patients, and presents treatment guidelines in a simple and interpretable format. It sets out the most appropriate steps in patient management at each therapy stage. Decision trees will be designed to give visual support to the care pathway. Because the treatment of IBD is rapidly changing and studies regularly provide new insights, the care pathway will be updated in IBD BeterKeten meetings after study closure.

\section{Implementation and adherence}

IBD specialists from IBD BeterKeten will safeguard implementation of the care pathway in their respective centres. They will be supported by a presentation of the working group to the care providers. To facilitate working according to the care pathway, we will implement the care pathway in electronic health records. Care providers will be able to schedule follow-up or diagnostics according to the care pathway with a single action. We will assess adherence to the care pathway by randomly sampling patients and comparing treatment decisions made for these patients with the treatment algorithms set out in the care pathway.

\section{Comparison}

The care pathway will be compared with current care by ways of the baseline measurement and adjustment for changes in the control group. All care providers continue their current practice according to their knowledge and local guidelines and treatment plans for the duration of the baseline measurement. The data collected in this period will give more insight into the current variation in practice, and can also be used to inform the design of the care pathway.

\section{Outcome}

To measure outcomes that matter to the patient, the standard set of patient-centred outcomes for IBD as defined by the International Consortium of Health Outcomes Measurement (ICHOM) will be used as the outcome measure of this study. ICHOM is an organisation that creates standard sets to measure the outcomes that matter most to patients. ${ }^{25}$ Patient-reported disease control as measured by the IBD-Control-8 score was chosen to serve as the primary outcome measure. This is a questionnaire that validly and reliably measures disease control from the patient perspective on a 16-point scale, and can distinguish between active disease and remission. ${ }^{36} 37$

The other outcomes from the standard set are secondary outcomes:

- IBD-attributable mortality.

- Remission, both clinician reported (biochemical, radiological, endoscopic and histologic) and patient reported (Manitoba IBD Index (MIBDI)) ${ }^{38}$

- Incidence of colorectal cancer.

- The presence of anaemia.

- Number of A\&E visits.

- Number and cumulative length of hospital admissions.
- Number of complications of any intervention for IBD.

- Long-term (>3 months) steroid use.

- The presence of fistulae symptoms.

- Body mass index as a proxy for nutritional status.

The MIBDI is a valid and patient-reported outcome measure, which can be used to classify disease activity on a dichotomous scale. The other outcomes from the standard set will be retrieved from the electronic health record. Other secondary outcomes are generic quality of life measured with the validated Patient-Reported Outcomes Measurement Information System - Global Health (PROMIS-GH) questionnaire, cost effectiveness and patient experience of care, using the Dutch Picker questionnaire. ${ }^{39} 40$

The cost-utility analysis (CUA) will be performed alongside the clinical study. In line with the recommendations of the National Healthcare Institute and the broad societal impact of IBD, the CUA will take a societal perspective. ${ }^{4142}$ Utility will be measured with the EQ-5D-5L (Dutch tariffs). ${ }^{43}$ The IBD-Control-8 score, which is more responsive to health state changes in IBD, will be used for an alternative cost-effectiveness analysis. ${ }^{36}$ Societal costs will be measured according to the guidelines of the National Healthcare Institute. ${ }^{42-44}$ Three types of societal costs are distinguished: healthcare costs, patient costs and other non-healthcare costs. For healthcare costs, primary care costs (primary care, home care and other out of hospital care) are distinguished from in-hospital costs (eg, number of admissions, MRIs and blood tests). Use of primary care will be measured using the shortened version of the Medical Consumption Questionnaire of the Institute of Medical Technology Assessment (iMTA). ${ }^{45}$ For healthcare use in secondary care, data will be collected from the electronic healthcare records. Productivity losses will be determined with the iMTA Productivity Cost Questionnaire. Measured productivity losses will be extrapolated from 1 month to 3 months. Absenteeism, presenteeism and lost unpaid work will be determined. Patient costs will be measured using a questionnaire on the following: travel costs; type, weeks and hours of informal care; insurance deductible; over the counter drug use; and other IBD-related costs. For all outcomes and their respective source, see table 1.

\section{Case mix}

To control for case mix differences between hospitals, we will collect the case mix variables defined in the ICHOM sets for risk adjustment for IBD care. ${ }^{25}$ Data will be collected on the following variables:

- Year of birth.

- Sex at birth.

- Education level as defined by UNESCO. ${ }^{46}$

- Smoking status

- Diagnosis (Crohn's disease, ulcerative colitis and indeterminate colitis)

- Year of diagnosis

- Disease phenotype according to the Montreal classification. $^{47}$ 
Table 1 Outcomes and their respective source

\begin{tabular}{|c|c|}
\hline Outcome & Source \\
\hline \multicolumn{2}{|l|}{ Primary } \\
\hline $\begin{array}{l}\text { Patient-reported disease } \\
\text { control }\end{array}$ & Patient-reported (IBD-Control) $)^{36} 37$ \\
\hline \multicolumn{2}{|l|}{ Secondary } \\
\hline IBD-attributable mortality & Chart review \\
\hline Clinical remission & Chart review \\
\hline $\begin{array}{l}\text { Endoscopic/radiologic } \\
\text { remission }\end{array}$ & Chart review \\
\hline Colorectal cancer & Chart review \\
\hline $\begin{array}{l}\text { Complications of IBD } \\
\text { treatment }\end{array}$ & Chart review \\
\hline Biochemical remission & Medical record \\
\hline Anaemia & Medical record \\
\hline A\&E visits & Medical record \\
\hline Hospital admissions & Medical record \\
\hline Long-term steroid use & Medical record \\
\hline Hospital costs & $\begin{array}{l}\text { Medical record and Dutch } \\
\text { reference prices } \\
52\end{array}$ \\
\hline Fistulae symptoms & Patient-reported \\
\hline BMI & Patient-reported \\
\hline Patient-reported remission & Patient-reported (MIBDI) ${ }^{38}$ \\
\hline Generic quality of life & Patient-reported (PROMIS-GH) ${ }^{39}$ \\
\hline Patient experience & Patient-reported (Picker) ${ }^{40}$ \\
\hline Utility & Patient-reported (EQ-5D-5L) ${ }^{4344}$ \\
\hline Primary care costs & $\begin{array}{l}\text { Patient-reported (iMCQ) }{ }^{45} \text { and } \\
\text { Dutch reference prices }\end{array}$ \\
\hline Productivity costs & $\begin{array}{l}\text { Patient-reported (iPCQ) }{ }^{57} \text { and } \\
\text { Dutch reference prices }\end{array}$ \\
\hline Patient costs & Patient-reported \\
\hline
\end{tabular}

IBD, inflammatory bowel disease; iMCQ, iMTA Medical Consumption Questionnaire; iMTA, Institute of Medical Technology Assessment; iPCQ, iMTA Productivity Cost Questionnaire; MIBDI, Manitoba IBD Index; PROMIS-GH, Patient-Reported Outcomes Measurement Information System Global Health.

- The presence of extraintestinal manifestations

- Medication use for IBD

- IBD-related surgery.

- Comorbidities as defined by the Self-administered Comorbidity Questionnaire (SCQ) with inclusion of some extra questions as defined by ICHOM. ${ }^{48}$

- Current or prior infection with tuberculosis, hepatitis B or HIV.

- Concomitant presence of primary sclerosing cholangitis.

- Treating hospital.

\section{Timing}

Patients can be included from 1 month before the start of the study (1 December 2020) until the end of the study (31 March 2023). Outcomes will be measured at the following time points as defined by ICHOM (see also tables 2 and 3). The IBD-Control, MIBDI, EQ-5D-5L and the PROMIS-10 will be administered when a participant is included in the study and at 6-month intervals from the start of the study. Cost questionnaires will be sent to patients at 3-month intervals from the start of the study. Demographics and comorbidity questionnaires will be sent at inclusion, at the start of the intervention period ( $\mathrm{t}=15$ months) and at the end of the study ( $\mathrm{t}=27$ months). Patient experience questionnaires will be distributed once a year after an outpatient clinic visit. To reduce questionnaire burden, some questionnaires at inclusion will not be sent if a patient is included 2 months (quality of life) or 3 months (case mix) before the respective questionnaires would be sent again.

Other outcomes will be retrieved from the electronic health records retrospectively, biannually and annually as recommended by ICHOM. A subset of the data (eg, age, gender, hospital healthcare use, anaemia, mortality and medication use) can be retrieved from the electronic health records anonymously. This data will be retrieved for the entire source population, as informed consent is not necessary for the use of anonymised data according to the Dutch law. This can be used to study possible selection bias.

\section{Statistical considerations \\ Power}

As our data are clustered longitudinally and per hospital, analytic sample size calculation is not appropriate. Thus, we used simulations to estimate power for different cluster sizes. The calculations were based on the following assumptions:

- A baseline IBD-Control score of 8 with an SD of $4 . .^{49} 50$

- Because of the clustering of data at two levels (within patients over time and patients clustered within hospitals), the degree of clustering has to be accounted for. As this is not reported in the literature, we estimated random effects for patients and hospitals with SDs between 0 and 4 (corresponding to intracluster correlation coefficients between 0 and $0.25)$.

- A change in IBD-Control score of 1 as clinically meaningful. Research has shown minimal important differences of $0.5 \mathrm{SD}$ for health-related quality of life instruments. However, as amelioration of a single symptom changes the score of the IBD-Control by $0.25 \mathrm{SD}$, we powered our study on this effect size.$^{51}$

The sample size calculation is further based on:

- Simulating data based on the assumptions listed above.

- Eight hospitals of between 1 and 50 patients each, in steps of 5;

- Ten thousand iterations per cluster size;

- Dropout of $10 \%$

- Type-1 error rate $(\alpha)$ of 0.05 two-sided;

- Power of at least $80 \%$ 
Table 2 Timing of questionnaires for patient included at or before $t=0 \mathrm{~m}$

\begin{tabular}{|c|c|c|c|c|c|c|c|c|}
\hline & Demographics & IBD-Control & MIBDI & SCQ & EQ-5D-5L/PROMIS-GH & iPCQ & iMCQ & Patient costs \\
\hline Om (study start) & $x$ & $x$ & $x$ & $x$ & $x$ & & & \\
\hline $3 \mathrm{~m}$ & & & & & & $x$ & $x$ & $X$ \\
\hline $9 \mathrm{~m}$ & & & & & & $x$ & $x$ & $x$ \\
\hline $12 m$ & & $x$ & $x$ & & $x$ & $x$ & $x$ & $x$ \\
\hline $18 m$ & & & & & & $x$ & $x$ & $x$ \\
\hline $21 \mathrm{~m}$ & & $\mathrm{X}$ & $\mathrm{X}$ & & $X$ & $\mathrm{X}$ & $\mathrm{X}$ & $\mathrm{X}$ \\
\hline $24 m$ & & & & & & $x$ & $x$ & $x$ \\
\hline $27 \mathrm{~m}$ & $X$ & $X$ & $\mathrm{X}$ & $X$ & $\mathrm{X}$ & $X$ & $X$ & $X$ \\
\hline
\end{tabular}

IBD, inflammatory bowel disease; iMCQ, iMTA Medical Consumption Questionnaire; iMTA, Institute of Medical Technology Assessment; IPCQ, iMTA Productivity Cost Questionnaire; m, month; MIBDI, Manitoba IBD Index; PROMIS-GH, Patient-Reported Outcomes Measurement Information System - Global Health; SCQ, Self-administered Comorbidity Questionnaire.

- Fitting a linear mixed effect model with random intercepts for patient and hospital and a fixed effect for intervention.

Power was defined as the number of iterations that found a statistically significant effect as a proportion of the total number of iterations. To account for our clustered data, 25 patients per hospital (a total of 200 patients) before the 6-month mark of the study would be required to have sufficient power $(>80 \%)$ to identify a change of 1 point of the IBD-Control score. We are striving to include all eligible patients to achieve a representative sample of the source population and to prevent selection bias.

Business intelligence (BI) departments in each of the participating hospitals will support patient screening and help reduce the logistic burden. The BI departments will use an algorithm to identify patients who meet the study inclusion criteria. These patients will receive a letter or an email from their hospital, asking for their consent to participate in the study. The algorithm will also identify the patient's care provider and next hospital visit. The care providers will be provided with this information to approach the patient for inclusion during the outpatient clinic visit. Patient recruitment should not be a time-consuming process, as the burden on the patient is low, the study is easy to explain and no randomisation or experimental treatment is used. Because all patients will receive an invitation letter to participate and care providers will remind them during their hospitals visit, we think that the minimum inclusion goal of 25 patients per hospital is feasible. Currently, 1001 patients have been included.

\section{Data analysis plan}

All missing data will be assessed whether these data are likely to be missing (completely) at random. If so, multivariate imputation by chained equations will be used to

Table 3 Timing of questionnaires for a patient included at $\mathrm{t}=10 \mathrm{~m}$

Demographics IBD-Control MIBDI SCQ EQ-5D-5L/PROMIS-GH iPCQ iMCQ Patient costs

$0 \mathrm{~m}$ (study start)
$3 \mathrm{~m}$
$6 \mathrm{~m}$
$9 \mathrm{~m}$

\begin{tabular}{|c|c|c|c|c|c|c|c|}
\hline $10 \mathrm{~m}$ (inclusion) & $x$ & & & & & & \\
\hline $12 \mathrm{~m}$ & & $X$ & $X$ & $\mathrm{X}$ & $X$ & $x$ & $x$ \\
\hline $15 \mathrm{~m}$ & $x$ & & & $x$ & $x$ & $x$ & $x$ \\
\hline $21 \mathrm{~m}$ & & $\mathrm{X}$ & $x$ & $\mathrm{X}$ & $x$ & $x$ & $x$ \\
\hline $24 m$ & & & & & $X$ & $X$ & $X$ \\
\hline
\end{tabular}

IBD, inflammatory bowel disease; iMCQ, iMTA Medical Consumption Questionnaire; iMTA, Institute of Medical Technology Assessment; iPCQ, iMTA Productivity Cost Questionnaire; MIBDI, Manitoba IBD Index; PROMIS-GH, Patient-Reported Outcomes Measurement Information System - Global Health; SCQ, Self-administered Comorbidity Questionnaire. 
impute missing data for variables used for adjustment. The primary outcome, IBD-Control-8 score, will be analysed on patient level using a linear mixed effects model of the form:

$$
Y_{i j t}=\beta_{0}+\eta_{j}+\theta_{i j}+\beta_{1} \iota+\beta_{t} v_{t}+\beta_{c} v_{c}+\varepsilon_{i j t}
$$

where $\mathrm{Y}$ is the IBD-Control-8 score $(0-16)$ of person $\mathrm{i}$ in cluster $\mathrm{j}$ at time $\mathrm{t}$ (0-6 months, 6-12 months, 15-21 months and 21-27 months); $\beta_{0}$ is the intercept; $\eta_{\mathrm{j}}$ is the cluster level random effect for cluster $\mathrm{j}$; $\theta_{\mathrm{ij}}$ is the patient level random effect for patient $i$ in cluster $j ; \beta_{1}$ the estimated difference between standard care $(l=0)$ and the care pathway $(\mathrm{l}=1) ; \beta_{\mathrm{t}}$ is a vector with coefficients for calendar time at the different time points $t$, captured as the vector $v_{t}$ with dummy variables for the different periods of follow-up; $\beta_{c}$ is a vector containing the coefficients for the case mix variables in the vector $v_{c}$ and $\varepsilon_{i \mathrm{jt}}$ is the residual error.

To adjust for case mix, we will use the variables from the ICHOM IBD set. These are age in years (continuous), sex at birth (dichotomous), education level (categorical: low, middle and high), smoking status (categorical: never, ex-smoker and current), comorbidities (SCQ and continuous), current or prior infection with tuberculosis (dichotomous), hepatitis B (dichotomous) and/ or HIV (dichotomous), diagnosis (categorical: Crohn's disease, ulcerative colitis and IBD-unknown/indeterminate), disease duration in years (continuous), phenotype according to the Montreal classification (for Crohn's disease: age of onset, localisation and behaviour and for ulcerative colitis and IBD-unknown/indeterminate: extension and all categorical), the presence of extraintestinal manifestations (categorical: none, skin, joint, hepatobiliary, eye and other) and concomitant presence of primary sclerosing cholangitis (categorical). The secondary outcomes from the ICHOM Standard Set will be analysed on patient level with a (generalised) linear mixed model of the same form as described above.

\section{Cost effectiveness}

As the standard of care and the new care pathway will be analysed for a 1-year period, this is also the time horizon for the CUA. No discounting of costs and effects will be applied to the 1-year period. Costs will be determined by multiplying measured healthcare use and productivity loss with reference prices or cost estimates in line with recommendations of the National Healthcare Institute. ${ }^{52} 53$ All costs will be transformed to the same year, adjusted for inflation using the Consumer Price Index, if necessary. The friction cost method will be used to estimate productivity costs. A sensitivity analysis using the human capital approach will also be performed.

To assess the cost effectiveness of the care pathway compared with usual care, crude and adjusted differences in costs and quality of life in the before and after groups from the regression models will be used to estimate the incremental cost-effectiveness ratio (ICER). Robustness of results will be evaluated using probabilistic sensitivity analysis (PSA) using Monte Carlo simulation. For the PSA, non-parametric bootstrapping with 2000 iterations will be used to determine uncertainty around the ICER. To support decision-making, calculation of the net monetary/health benefits at the relevant willingness to pay levels, acceptability curves and value of information analysis will be added.

\section{Variation}

To assess the variation in outcomes and costs between hospitals, the intraclass correlation coefficient (ICC) will be used. The ICC is defined as:

$$
\operatorname{ICC}(\text { Cluster })=\frac{\sigma_{\eta}^{2}}{\sigma_{\eta}^{2}+\sigma_{\theta}^{2}+\sigma_{\varepsilon}^{2}}
$$

which can be interpreted as the variance explained by the hospital as a proportion of the total variance. For the baseline period, data will be analysed using the aforementioned mixed effects models omitting the coefficient for the care pathway.

To assess the effect of the care pathway on variation, data from the six hospitals that implemented the care pathway will be analysed for the two periods using the aforementioned mixed effects model, without the coefficient for the care pathway. This model will be compared with a model that estimates a random effect per hospital for the baseline period and the care pathway period separately. The effect of the care pathway on variation will then be formally tested using a likelihood-ratio test comparing the two models.

\section{Patient and public involvement}

Crohn \& Colitis NL (Dutch Crohn's and Colitis Patient Organisation) collaborated in the design of this study. They critically revised the study design and helped in piloting the questionnaires. They will be involved in the working group that is responsible for the development of the care pathway.

\section{ETHICS AND DISSEMINATION}

The study was deemed to not be subject to the Wet medisch-wetenschappelijk onderzoek met mensen (WMO; Medical Research Involving Human Subjects Act) by the Medical Ethics Committee of the Erasmus MC, the Netherlands (registration number: MEC-2020-075). The study is not subject to the WMO as the implementation of the care pathway is a change in the local standard of care, patients are not randomised to different treatment groups and patients do not undergo invasive procedures for the study. Informed consent for questionnaires and chart review will be obtained by local investigators (online supplemental file 2). Data of all participating centres will be collected using electronic case report forms and entered in Castor EDC, an electronic database that is ISO 27001 certified.$^{54}$ Data will be coded and handled based on the General Data Protection Regulation (GDPR). A data monitoring committee is not necessary, as the intervention under study is a change in the standard of care. 
The principal investigators and study coordinator will have access to the final dataset. The dataset will be available on reasonable request. The study team is responsible for data analysis and reporting. Results will be fed back to participating centres and disseminated through peer-reviewed journals and presented at (inter)national conferences. The study team will make the decision to publish, and the funder and sponsor had and will have no influence on the research question, study design, data collection or analysis, or decision to publish.

\section{DISCUSSION}

The IBD Value study aims to assess the effect of a care pathway for patients with IBD treated with biologicals on health outcomes and cost effectiveness as compared with current care. The study design is a longitudinal multicentre non-randomised parallel cluster trial with a baseline period. This design was chosen because the care pathway is an intervention on hospital level making a patient level study infeasible. A randomised cluster trial was logistically not possible as the care pathway will be developed by the six intervention hospitals and they can, therefore, not be blinded to the intervention. A randomised stepped wedge cluster trial would run into problems with contamination of the control period as the care pathway would need to be developed before the first clusters moved to the intervention group. This would lead to providers from the control cluster not being blinded to the intervention as they would be in the working group.

Strengths of this study are the baseline period and control group, as well as blinding of the control group. The baseline period and control group make it possible to control for time trends, such as a change in practice over time, when analysing the effect of the care pathway. By comparing the change in outcomes of the intervention group with the change in the control group, it is possible to distinguish the effect of the care pathway from time trends that impact outcomes or costs. A present-day example would be the impact of the COVID-19 pandemic on IBD healthcare. ${ }^{55}$ Blinding of the control group to the intervention prevents contamination of the control group. If not blinded to the intervention, the control group could (subconsciously) change standard of care to incorporate the care pathway, and bias the effect of the care pathway towards the null.

The main weakness of our study design is the lack of randomisation. As hospitals are allocated to the intervention and control groups non-randomly, there might be differences in confounders both on the cluster and patient level. Even though we correct for the average trend in outcomes or costs, there might be residual confounding because of systematic differences in hospitals between the intervention and control groups. Confounding at the patient level can occur because of differences in case mix between the intervention and control group. To reduce bias, we will control for case mix variables at the patient level as specified in the ICHOM IBD Standard Set. ${ }^{25}$
The main challenge of our study is implementation of and adherence to the intervention. To effectively implement the care pathway, we will take several steps during the design and implementation phases. ${ }^{56}$ First, the care pathway will be developed by a mixed group of stakeholders to ensure involvement of all hospitals and patients. Second, the care pathway was adjusted to the local context as to not disrupt local processes. Third, implementation of the care pathway in the participating hospitals will be done by the respective IBD specialists to ensure support from the rest of the medical staff. Fourth, the care pathway will be supported in the electronic health records to reduce burden on physicians and nurses. Last, adherence to the care pathway will be reported to the participating hospitals to evaluate implementation and detect potential barriers for implementation.

\section{Author affiliations}

${ }^{1}$ Department of Gastroenterology and Hepatology, Franciscus Gasthuis en Vlietland, Rotterdam, The Netherlands

${ }^{2}$ Department of Gastroenterology and Hepatology, Erasmus Medical Center, Rotterdam, The Netherlands

${ }^{3}$ Department of Public Health, Erasmus Medical Center, Rotterdam, The Netherlands ${ }^{4}$ Department of Statistics and Education, Franciscus Gasthuis en Vlietland,

Rotterdam, The Netherlands

${ }^{5}$ Department of Genetics, University of Groningen, Groningen, The Netherlands

${ }^{6}$ Crohn \& Colitis NL, Woerden, The Netherlands

${ }^{7}$ Department of Gastroenterology and Hepatology, Maasstad Hospital, Rotterdam, The Netherlands

${ }^{8}$ Department of Gastroenterology and Hepatology, Albert Schweitzer Ziekenhuis, Dordrecht, The Netherlands

Acknowledgements Conny Nuis and Daniëlle van der Horst for their assistance in designing the care pathway.

Collaborators Centres and lead investigators that collaborate as the Southwest Netherlands IBD Study Group: Department of Gastroenterology, Albert Schweitzer Hospital, Dordrecht: Vincent de Jonge and Frank Wolfhagen; Department of Gastroenterology, Amphia Hospital, Breda: Alexander Bodelier and Jerome Sint Nicolaas; Department of Gastroenterology, Erasmus University Medical Center, Rotterdam: Janneke van der Woude and Annemarie de Vries; Department of Gastroenterology, Franciscus Gasthuis \& Vlietland, Rotterdam and Schiedam: Rachel West, Desirée van Noord and Reinier van Linschoten; Department of Gastroenterology, Maasstad Hospital, Rotterdam: Evelyne Verweij and François Kubben; Department of Gastroenterology, IJsselland Hospital, Capelle aan den IJssel: Claire Fitzpatrick and Kristin Robbers; Department of Gastroenterology, Ikazia Hospital, Rotterdam: Hestia Vermeulen;Department of Gastroenterology, Reinier de Graaf Gasthuis, Delft: Sanne van der Wiel and Sita Jansen.

Contributors RCAvL, DvN and RLW designed the study. NvL provided epidemiological expertise. DN provided statistical expertise. EB provided expertise in economic evaluation during the trial design. MS, CJvdW and JAH critically reviewed the study design. CJvdW, RLW, KEV, VdJ and RCAvL participated in the design of the intervention. RCAvL drafted the manuscript and ensures daily study management as study coordinator. All authors read, critically revised and approved the final manuscript. DvN and RLW are the principal investigators and share last authorship.

Funding This study is funded partly by the Research and Development Foundation (grant number: N/A) of the Franciscus Gasthuis \& Vlietland, the Franciscus Research Foundation (grant number: N/A), AbbVie, Falk, Ferring, Janssen, MSD, Pfizer and Takeda. This study is sponsored by Franciscus Gasthuis \& Vlietland, Rotterdam, the Netherlands.

Competing interests RCAvL, NvL, DN, EB, MS, VdJ, KEV and JAH has nothing to disclose. CJvdW reports grants from Pfizer and Janssen and personal fees from AbbVie and Celltrion outside the submitted work. DvN reports personal fees from Janssen and Takeda outside the submitted work. RLW reports personal fees from AbbVie, Janssen and Pfizer outside the submitted work. 
Patient consent for publication Not applicable.

Provenance and peer review Not commissioned; externally peer reviewed.

Data availability statement Data are available upon reasonable request. The dataset generated in this study will be available on reasonable request.

Supplemental material This content has been supplied by the author(s). It has not been vetted by BMJ Publishing Group Limited (BMJ) and may not have been peer-reviewed. Any opinions or recommendations discussed are solely those of the author(s) and are not endorsed by BMJ. BMJ disclaims all liability and responsibility arising from any reliance placed on the content. Where the content includes any translated material, BMJ does not warrant the accuracy and reliability of the translations (including but not limited to local regulations, clinical guidelines, terminology, drug names and drug dosages), and is not responsible for any error and/or omissions arising from translation and adaptation or otherwise.

Open access This is an open access article distributed in accordance with the Creative Commons Attribution Non Commercial (CC BY-NC 4.0) license, which permits others to distribute, remix, adapt, build upon this work non-commercially, and license their derivative works on different terms, provided the original work is properly cited, appropriate credit is given, any changes made indicated, and the use is non-commercial. See: http://creativecommons.org/licenses/by-nc/4.0/.

\section{ORCID iD}

Reinier Cornelis Anthonius van Linschoten http://orcid.org/0000-0003-3052-596X

\section{REFERENCES}

1 Magro F, Rodrigues A, Vieira Al, et al. Review of the disease course among adult ulcerative colitis population-based longitudinal cohorts. Inflamm Bowel Dis 2012;18:573-83.

2 Peyrin-Biroulet L, Loftus EV, Colombel J-F, Colombel J-F, et al. The natural history of adult Crohn's disease in population-based cohorts. Am J Gastroenterol 2010;105:289-97.

3 Bernstein CN, Blanchard JF, Rawsthorne P, et al. The prevalence of extraintestinal diseases in inflammatory bowel disease: a populationbased study. Am J Gastroenterol 2001;96:1116-22.

4 Isaacs KL. How prevalent are extraintestinal manifestations at the initial diagnosis of IBD? Inflamm Bowel Dis 2008;14:S198-9.

5 Levine JS, Burakoff R. Extraintestinal manifestations of inflammatory bowel disease. Gastroenterol Hepatol 2011;7:235.

6 Borren NZ, van der Woude CJ, Ananthakrishnan AN. Fatigue in IBD: epidemiology, pathophysiology and management. Nat Rev Gastroenterol Hepatol 2019;16:247-59.

7 Knowles SR, Graff LA, Wilding $\mathrm{H}$, et al. Quality of life in inflammatory bowel disease: a systematic review and meta-analyses-part I. Inflamm Bowel Dis 2018;24:742-51.

8 van Linschoten RCA, Visser E, Niehot CD, et al. Systematic review: societal cost of illness of inflammatory bowel disease is increasing due to biologics and varies between continents. Aliment Pharmacol Ther 2021;54:234-48.

9 Feagan BG, Reilly MC, Gerlier L, et al. Clinical trial: the effects of certolizumab pegol therapy on work productivity in patients with moderate-to-severe Crohn's disease in the precise 2 study. Aliment Pharmacol Ther 2010;31:1276-85.

10 Lichtiger S, Binion DG, Wolf DC, et al. The choice trial: adalimumab demonstrates safety, fistula healing, improved quality of life and increased work productivity in patients with Crohn's disease who failed prior infliximab therapy. Aliment Pharmacol Ther 2010;32:1228-39.

11 Feagan BG, Sandborn WJ, Lazar A, et al. Adalimumab therapy is associated with reduced risk of hospitalization in patients with ulcerative colitis. Gastroenterology 2014;146:110-8.

12 Costa J, Magro F, Caldeira D, et al. Infliximab reduces hospitalizations and surgery interventions in patients with inflammatory bowel disease: a systematic review and meta-analysis. Inflamm Bowel Dis 2013;19:2098-110.

13 Spiegel BMR, Ho W, Esrailian E, et al. Controversies in ulcerative colitis: a survey comparing decision making of experts versus community Gastroenterologists. Clin Gastroenterol Hepatol 2009;7:168-74.

14 Ananthakrishnan AN, Kwon J, Raffals L, et al. Variation in treatment of patients with inflammatory bowel diseases at major referral centers in the United States. Clin Gastroenterol Hepatol 2015;13:1197-200.

15 Esrailian E, Spiegel BMR, Targownik LE, et al. Differences in the management of Crohn's disease among experts and community providers, based on a national survey of sample case vignettes. Aliment Pharmacol Ther 2007;26:1005-18.

16 Singh S, Chowdhry M, Umar S, et al. Variations in the medical treatment of inflammatory bowel disease among Gastroenterologists. Gastroenterol Rep 2018;6:61-4.

17 Samaan MA, Arkir Z, Ahmad T, et al. Wide variation in the use and understanding of therapeutic drug monitoring for anti-TNF agents in inflammatory bowel disease: an inexact science? Expert Opin Biol Ther 2018;18:1271-9.

18 Bezzio C, Imperatore N, Armuzzi A, et al. Barriers to anti-TNFalpha prescription among Italian physicians managing inflammatory bowel disease. GastroHep 2019:1:93-9.

19 Weaver KN, Kappelman MD, Sandler RS, et al. Variation in care of inflammatory bowel diseases patients in Crohn's and colitis foundation of America partners: role of gastroenterologist practice setting in disease outcomes and quality process measures. Inflamm Bowel Dis 2016;22:2672-7.

20 Jeuring SFG, Bours PHA, Zeegers MP, et al. Disease outcome of ulcerative colitis in an era of changing treatment strategies: results from the Dutch population-based IBDSL cohort. J Crohns Colitis 2015;9:837-45

21 Jeuring SFG, van den Heuvel TRA, Liu LYL, et al. Improvements in the long-term outcome of Crohn's disease over the past two decades and the relation to changes in medical management: results from the population-based IBDSL cohort. Am J Gastroenterol 2017;112:325-36.

22 Murthy SK, Begum J, Benchimol El, et al. Introduction of antiTNF therapy has not yielded expected declines in hospitalisation and intestinal resection rates in inflammatory bowel diseases: a population-based interrupted time series study. Gut 2020;69:274-82.

23 Porter ME. What is value in health care? N Engl J Med 2010;363:2477-81

24 Lehtonen L, Wild C, Ricciardi W. Defining Value in "Value-Based Healthcare". In: Commission E, ed. Report of the Expert Panel on effective ways of investing in Health (EXPH). Luxembourg: European Commission, 2019

$25 \mathrm{Kim} \mathrm{AH}$, Roberts C, Feagan BG, et al. Developing a standard set of patient-centred outcomes for inflammatory bowel diseasean international, cross-disciplinary consensus. J Crohns Colitis 2018;12:408-18.

26 Porter ME, Larsson S, Lee TH. Standardizing patient outcomes measurement. N Engl J Med 2016;374:504-6.

27 van Deen WK, Spiro A, Burak Ozbay A, et al. The impact of valuebased healthcare for inflammatory bowel diseases on healthcare utilization: a pilot study. Eur J Gastroenterol Hepatol 2017;29:331-7.

28 Lewin SM, McConnell RA, Patel R, et al. Improving the quality of inpatient ulcerative colitis management: promoting evidence-based practice and reducing care variation with an inpatient protocol. Inflamm Bowel Dis 2019;25:1822-7.

29 Sack C, Phan VA, Grafton R, et al. A chronic care model significantly decreases costs and healthcare utilisation in patients with inflammatory bowel disease. J Crohns Colitis 2012;6:302-10.

30 Panella M, Marchisio S, Di Stanislao F. Reducing clinical variations with clinical pathways: do pathways work? Int J Qual Health Care 2003;15:509-21.

31 Chan A-W, Tetzlaff JM, Gøtzsche PC, et al. Spirit 2013 explanation and elaboration: guidance for protocols of clinical trials. BMJ 2013;346:e7586.

32 IBD BeterKeten. IBD-zorg; gedeelde zorg 2020. Available: http:// www.ibd-zorg.nl/

33 Nederlandse Vereniging van Maag-Darm-Leverartsen. Handleiding Behandeling IBD - 2014-2015: Moderniseren van de Richtlijn IBD 2009: Nederlandse Vereniging van Maag-Darm-Leverartsen, 2015.

34 Torres J, Bonovas S, Doherty G, et al. ECCO guidelines on therapeutics in Crohn's disease: medical treatment. J Crohns Colitis 2020;14:4-22.

35 Harbord M, Eliakim R, Bettenworth D, et al. Third European evidence-based consensus on diagnosis and management of ulcerative colitis. Part 2: current management. J Crohns Colitis 2017;11:769-84.

36 Bodger K, Ormerod C, Shackcloth D, et al. Development and validation of a rapid, generic measure of disease control from the patient's perspective: the IBD-control questionnaire. Gut 2014;63:1092-102.

37 de Jong ME, Taal E, Thomas PWA, et al. Cross-cultural translation and validation of the IBD-control questionnaire in the Netherlands: a patient-reported outcome measure in inflammatory bowel disease. Scand J Gastroenterol 2021;56:155-61.

38 Clara I, Lix LM, Walker JR, et al. The Manitoba IBD index: evidence for a new and simple indicator of IBD activity. Am J Gastroenterol 2009;104:1754-63. 
39 Hays RD, Bjorner JB, Revicki DA, et al. Development of physical and mental health summary scores from the patient-reported outcomes measurement information system (PROMIS) global items. Qual Life Res 2009;18:873-80.

40 Bastemeijer CM, Boosman $\mathrm{H}$, Zandbelt L, et al. Patient experience monitor (Pem): the development of new short-form Picker experience questionnaires for hospital patients with a wide range of literacy levels. Patient Relat Outcome Meas 2020;11:221-30. Volume.

41 van der Valk ME, Mangen M-JJ, Leenders M, et al. Healthcare costs of inflammatory bowel disease have shifted from hospitalisation and surgery towards anti-TNF $\alpha$ therapy: results from the coin study. Gut 2014;63:72-9.

42 Hakkaart-van Roijen L, van der Linden N, Bouwmans C. Richtlijn voor het uitvoeren van economische evaluaties in de gezondheidszorg. In: Authority DH, ed. Dutch healthcare authority, 2016: 1-73. https://www.zorginstituutnederland.nl/over-ons/ publicaties/publicatie/2016/02/29/richtlijn-voor-het-uitvoeren-vaneconomische-evaluaties-in-de-gezondheidszorg

$43 \mathrm{M}$ Versteegh M, M Vermeulen K, M A A Evers S, et al. Dutch tariff for the five-level version of EQ-5D. Value Health 2016;19:343-52.

44 Herdman M, Gudex C, Lloyd A, et al. Development and preliminary testing of the new five-level version of EQ-5D (EQ-5D-5L). Qual Life Res 2011;20:1727-36.

45 iMTA Productivity and Health Research Group. Handleiding iMTA medical cost questionnaire (iMCQ. Rotterdam: MTA, Erasmus Universiteit Rotterdam, 2018. www.imta.nl

46 UNESCO Institute for Statistics. International standard classification of education: ISCED 2011: UNESCO Institute for Statistics Montreal 2012.

47 Satsangi J, Silverberg MS, Vermeire S, et al. The Montreal classification of inflammatory bowel disease: controversies, consensus, and implications. Gut 2006;55:749-53.
48 Sangha $\mathrm{O}$, Stucki G, Liang $\mathrm{MH}$, et al. The self-administered comorbidity questionnaire: a new method to assess comorbidity for clinical and health services research. Arthritis Rheum 2003;49:156-63.

49 Mikocka-Walus A, Massuger W, Knowles SR. Quality of care in inflammatory bowel disease: actual health service experiences fall short of the Standards. J Intern Med 2019.

50 Bodger K, Gledhill T, Driscoll R. PWE-054 independent validation of the ibd-control questionnaire: results from a large-scale electronic patient experience survey (ibd2020). BMJ Publishing Group, 2015.

51 Norman GR, Sloan JA, Wyrwich KW. Interpretation of changes in health-related quality of life: the remarkable universality of half a standard deviation. Med Care 2003;41:582-92.

52 Hakkaart-van Roijen L, Van der Linden N, Bouwmans C. Kostenhandleiding. Methodologie van kostenonderzoek en referentieprijzen voor economische evaluaties in de gezondheidszorg. Zorginstituut Nederland, 2015.

53 National Health Care Institute. Medicijnkosten 2019. Available: https://www.medicijnkosten.nl/ [Accessed 18-06-2019].

54 Castor Electronic Data Capture [program]. Amsterdam 2016.

55 Te Groen M, Derks MEW, Kuijpers CCHJ, et al. Reduction in inflammatory bowel disease healthcare during the coronavirus disease 2019 pandemic: a nationwide retrospective cohort study. Gastroenterology 2021;160:935-7.

56 Evans-Lacko S, Jarrett M, McCrone P, et al. Facilitators and barriers to implementing clinical care pathways. BMC Health Serv Res 2010;10:182.

57 Bouwmans C, Krol M, Severens $\mathrm{H}$, et al. The iMTA productivity cost questionnaire: a standardized instrument for measuring and Valuing health-related productivity losses. Value Health 2015;18:753-8. 